\title{
Dwarf irregular galaxy Leo A extends even farther, according to HST WFC3 photometry ${ }^{\star}$
}

\author{
R. Stonkutè ${ }^{1,2}$, M. Čeponis ${ }^{1}$, A. Leščinskaite ${ }^{1}$, R. Naujalis ${ }^{1}$, and V. Vansevičius ${ }^{1,2}$ \\ 1 Center for Physical Sciences and Technology, Saulètekio av. 3, 10257 Vilnius, Lithuania \\ e-mail: vladas.vansevicius@ff.vu.lt \\ 2 Vilnius University Observatory, Čiurlionio 29, 03100 Vilnius, Lithuania
}

Received 28 August 2017 / Accepted 18 January 2018

\begin{abstract}
Context. Leo A is an isolated gas-rich dwarf irregular galaxy of low stellar mass and metallicity residing at the outskirts of the Local Group. Ages of the stellar populations in Leo A range from $\sim 10 \mathrm{Myr}$ to $\sim 10 \mathrm{Gyr}$. A large stellar halo (up to the deprojected distance of $\sim 1.7 \mathrm{kpc}$ ) was discovered based on Subaru wide-field photometry of red giant branch stars.

Aims. We aim to study stellar populations at the very outer region of the Leo A galaxy.

Methods. We analysed HST WFC3 archive observation data in the field that is partly located beyond the previously known limits of the Leo A galaxy. We performed photometry of star-like objects in the $F 475 W$ and $F 814 W$ passbands and studied the spatial distribution of the Leo A stars below the horizontal branch.

Results. We report a discovery of stellar halo populations (subgiants and faint red giants) in the Leo A galaxy extending up to $\sim 2.3 \mathrm{kpc}$ deprojected distance from the galaxy centre. Analysis of the colour-magnitude diagram suggests old ( $>5$ Gyr) stellar populations of very low metallicity $(Z \sim 0.0001)$.
\end{abstract}

Key words. galaxies: dwarf - galaxies: stellar content - galaxies: individual: Leo A (DDO 69)

\section{Introduction}

Dwarf irregular galaxies are assumed to be simple gas-rich stellar systems, presumably the "building blocks" of large galaxies (Tosi 2003). An analysis of these systems, which are likely built up solely through self-enrichment, could provide insight into the early star formation history in the Universe.

Leo A (Fig. 1) is an isolated dwarf irregular galaxy in the Local Group and could serve as an example of such a building block. It is a gas-rich (Young \& Lo 1996; Hunter et al. 2012) stellar system dominated by dark matter (Brown et al. 2007; Kirby et al. 2017) of low stellar mass (Cole et al. 2007) and low metallicity (van Zee et al. 2006; Kirby et al. 2017). It consists of multiple stellar populations that are between $\sim 10 \mathrm{Myr}$ to $\sim 10$ Gyr old. The present-day star formation activity is traced by $\mathrm{H}_{\text {II }}$ regions, while the existence of an old stellar population is proved by the detection of RR Lyr stars (Dolphin et al. 2002; Bernard et al. 2013). Detailed studies of stellar content in Leo A were performed with the Hubble Space Telescope (HST), which was equipped with the Wide Field and Planetary Camera 2 (WFPC2; Tolstoy et al. 1998; Schulte-Ladbeck et al. 2002) and with the Advanced Camera for Surveys (ACS; Cole et al. 2007), by imaging of the central part.

The importance of stellar populations in the galaxy outskirts for studying galaxy build-up and star formation processes is well recognized and has been widely discussed recently (Gil de Paz et al. 2017; Knapen et al. 2017). An especially important point to address in the discussion of gas-rich

\footnotetext{
* Photometric data are only available at the CDS via anonymous ftp to cdsarc.u-strasbg.fr $(130.79 .128 .5)$ or via http://cdsarc.u-strasbg.fr/viz-bin/qcat?]/A+A/614/A144
}

dwarf galaxy evolution scenarios is the ratio of the sizes of their stellar and Hi components. Wide photometric mapping of the stellar component was performed with the Subaru Telescope, which was equipped with the Suprime-Cam mosaic camera (Stonkute et al. 2014). We analysed the distribution of red giant branch (RGB) stars, but they are not numerous at the very outskirts of the galaxy, and in the colour-magnitude diagram (CMD), they overlap with the Milky Way (MW) foreground stars. Therefore, a correct determination of the Leo A size based solely on RGB stars is a rather difficult task. To overcome this problem, we decided to study stellar populations below the horizontal branch by employing available archive HST Wide Field Camera 3 (WFC3/UVIS) observations.

The following basic parameters of the Leo A galaxy as derived from the RGB star distribution (Vansevičius et al. 2004) are adopted in this study: (1) centre coordinates of the galaxy, $\alpha=9^{\mathrm{h}} 59^{\mathrm{m}} 24^{\mathrm{s}}, \delta=+30^{\circ} 44^{\prime} 47^{\prime \prime}(\mathrm{J} 2000)$; (2) ellipticity - ratio of the semi-minor to the semi-major axis, $b / a=0.6$; (3) position angle of the major axis, $\mathrm{PA}=114^{\circ}$; and (4) the semi-major axis, $a=7.5^{\prime}$. We also assumed the Holmberg semi-major axis, $a=3.5^{\prime}$ (Mateo 1998) and the semi-major axis of the Hi envelope, $a=7.5^{\prime}$ (Young \& Lo 1996). The distance to Leo A is assumed to be $800 \mathrm{kpc}\left(1^{\prime} \approx 230 \mathrm{pc}\right.$; Dolphin et al. 2002). The foreground extinction towards Leo $\mathrm{A}$ is derived from the extinction maps (Schlafly \& Finkbeiner 2011), $A(F 475 W)=0.067$ and $A(F 814 W)=0.032$, assuming the standard extinction law of the Milky Way with $R_{V}=3.1$ (Fitzpatrick 1999).

The structure of the paper is the following: Sect. 2 presents details of the archive observation data and reductions. Sect. 3 presents results and a brief discussion. Conclusions are presented in Sect. 4. 


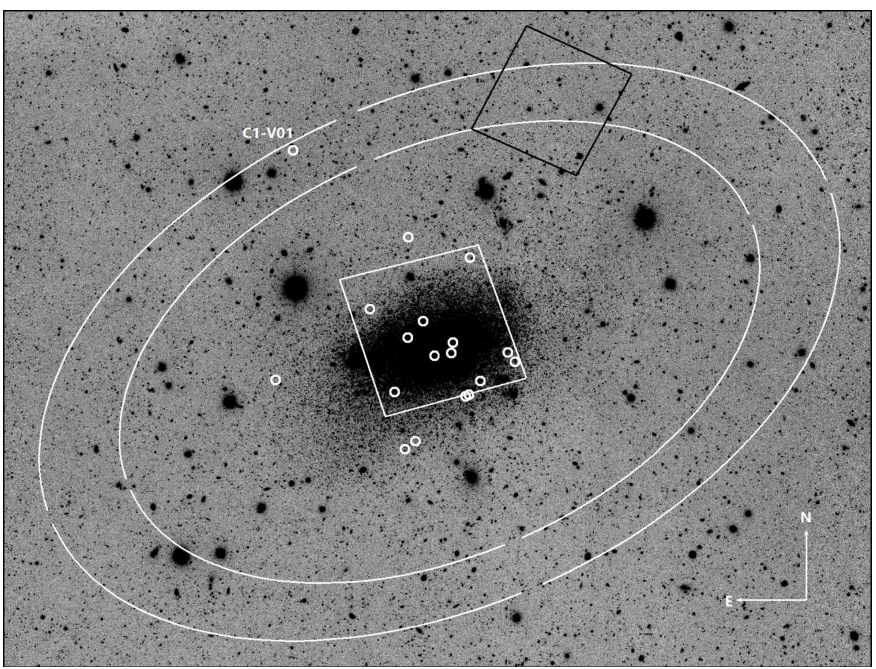

Fig. 1. Subaru Suprime-Cam $B$-passband image of the Leo A galaxy (Stonkute et al. 2014). The ellipses (semi-minor to semimajor axis ratio: $b / a=0.6 ; \mathrm{PA}=114^{\circ}$ ) of $8^{\prime}$ and $10^{\prime}$ along the semi-major axis, centred at $\alpha=9^{\mathrm{h}} 59^{\mathrm{m}} 24^{\mathrm{s}}, \delta=$ $+30^{\circ} 44^{\prime} 47^{\prime \prime}$ (J2000), are shown. The HST fields, marked white (ACS) and black (WFC3), are taken from the HST Proposal 12273 by Roeland van der Marel (https://archive.stsci.edu/proposal_ search.php?mission=hst\&id=12273). The RR Lyr stars discovered by Dolphin et al. (2002) and Bernard et al. (2013) are marked by white open circles. North is up, east is left.

\section{Observations and data reductions}

We used HST WFC3 $F 475 W$ and $F 814 W$ passband observation data of the Leo A galaxy obtained for the HST Proper Motion Collaboration (HSTPMC) program - Mass of the Local Group from Proper Motions of Distant Dwarf Galaxies (the HST proposal GO 12273, the principal investigator Roeland P. van der Marel) during the HST cycle 18 on December 28, 2011, in parallel field WFC3/UVIS.

The WFC3/UVIS camera was pointed $\sim 6^{\prime}$ away $(\mathrm{N}-\mathrm{W})$ from the Leo A galaxy centre in the outer halo (Fig. 1). A total exposure time of 5408 and 5282 seconds was obtained in passbands $F 475 W$ and $F 814 W$, respectively. The archival data were downloaded from the Mikulski Archive for Space Telescopes (MAST). We retrieved bias-subtracted, flat-fielded, charge transfer efficiency (CTE) corrected WFC3/UVIS flc images produced by the STScI On-The-Fly-Reprocessing (OTFR) pipeline OPUS versions 2016_2, which used CALWF3 version 3.4.

To perform stellar photometry, we used the software package DOLPHOT 2.0 (Dolphin 2000, and many unpublished updates). We followed the recommended preprocessing steps and the photometry recipe provided in the manual for the WFC3 module (version April 17, 2016). We used DrizzlePac 2.1.6 (default parameter values) to create clean, deep-drizzled reference frames for object detection and coordinate transformations from four sub-exposures in each of the $F 475 W$ and $F 814 W$ passbands. This also allowed us to flag the cosmic rays in the individual flc images and update data quality images.

The HST WFC3/UVIS field is located at the uncrowded outskirts of the Leo A galaxy. Therefore, we used values of DOLPHOT parameters recommended in the WFC3 manual for uncrowded fields: the FitSky parameter was set to 1, which means the sky fitting in an annulus around each star $\left(R_{\text {inner }}=15\right.$, $R_{\text {outer }}=35$ pixels) and point-spread function (PSF) fitting inside a radius of $R_{\text {apert }}=4$ pixels.
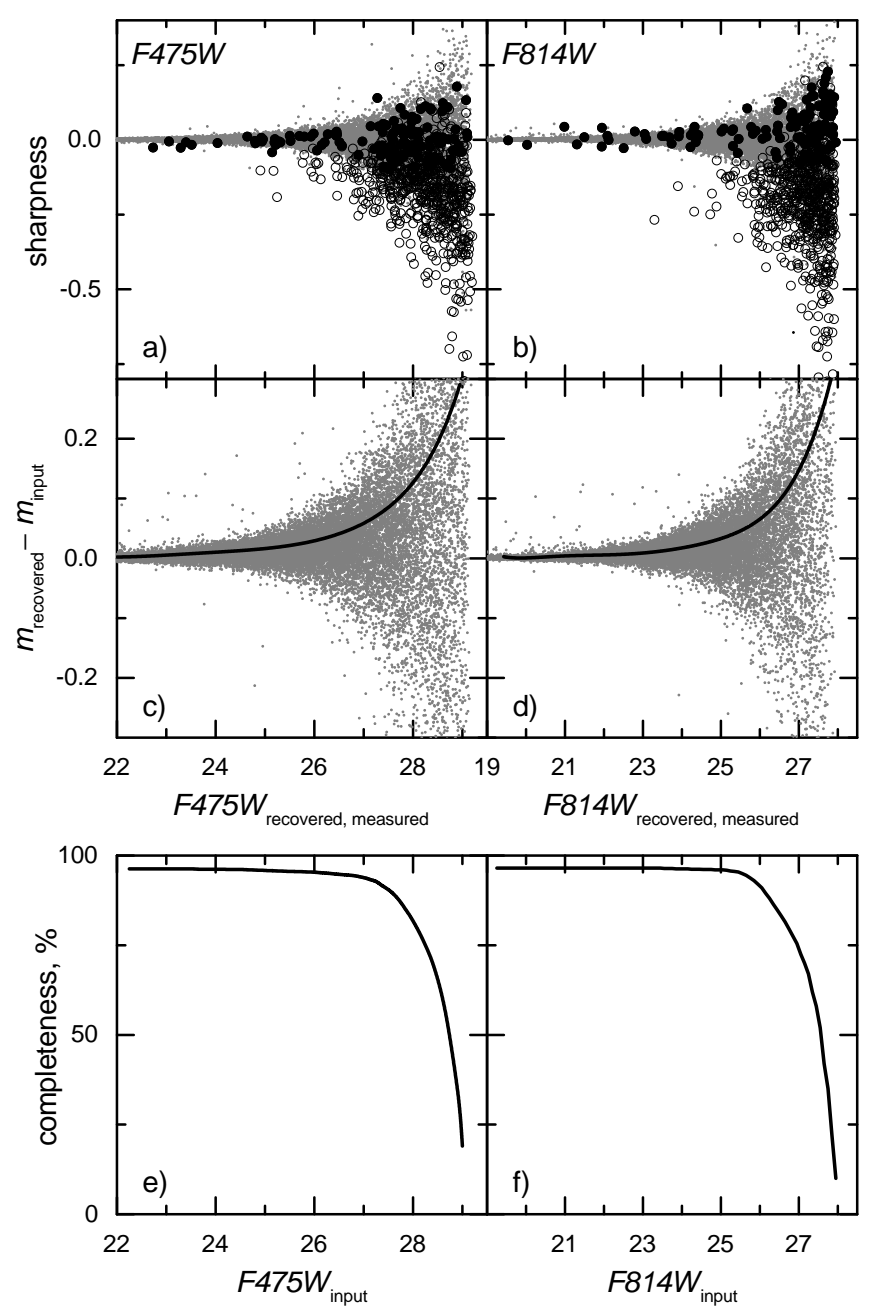

Fig. 2. AST results in the $F 475 W$ and $F 814 W$ HST WFC3 passbands. Panels $a$ and $b$ : grey dots show the sharpness of the AST stars; filled (star-like objects) and open (extended objects) circles show the sharpness of the real measured objects. Panels $c$ and $d$ : grey dots indicate the difference of recovered and input magnitudes of the AST stars; black lines indicate average photometric errors provided by DOLPHOT for real objects. Results in panels $a-d$ are shown vs. recovered and measured magnitudes. Derived photometry completeness estimates in the $F 475 W$ and $F 814 W$ passbands are shown in panels $e$ and $f$ vs. input magnitudes, respectively.

DOLPHOT determines magnitudes, magnitude errors, object fit, and shape parameters in individual flc frames, and then combines them for each filter. To combine the magnitudes, we set a parameter FlagMask $=7$, which means that only measurements with error flags equal to zero were used.

Numerous extended objects in the WFC3 field can hamper the photometry of nearby stars, therefore setting the DOLPHOT parameter Force $1=\theta$ could result in a false cleaning of actual stars from the photometric catalogue. Since the CMD is needed to be as complete as possible for our study, we set the parameter Force $1=1$, which forced all detected sources to be fitted as stars. This resulted in a strongly contaminated CMD, as hot pixels and extended objects were also measured as single stars.

The output photometry file had measurements of more than 60000 objects. In order to discard non-point sources and measurements of artefacts, the photometry catalogue was cleaned. We rejected objects that had measurements in only one filter or had a measured DOLPHOT photometry magnitude error 


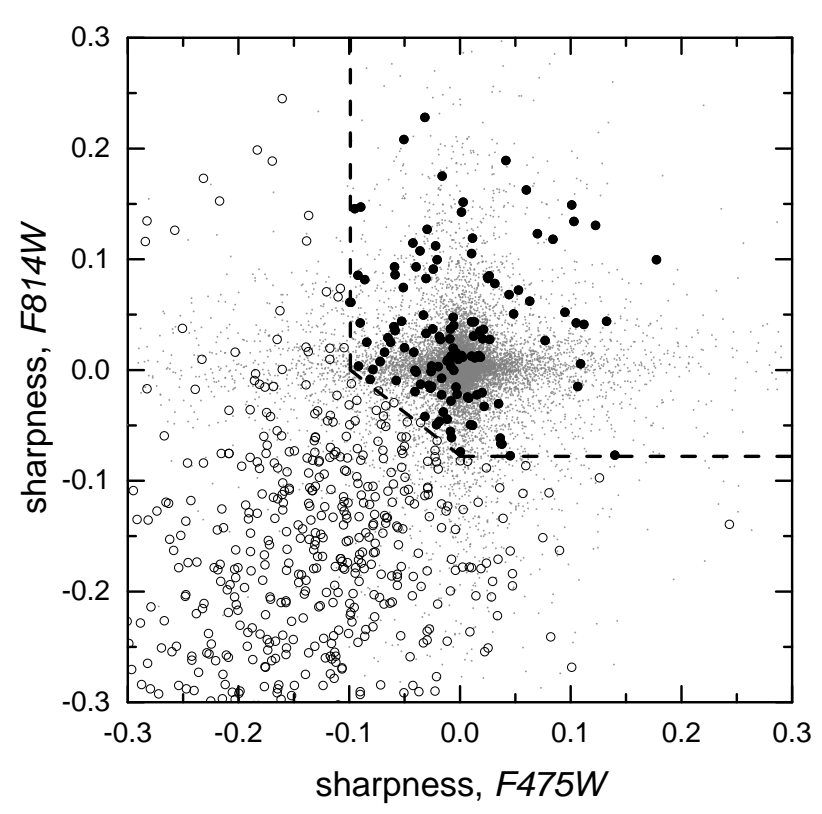

Fig. 3. Sharpness in the $F 475 W$ and $F 814 W$ HST WFC3 passbands of the measured point-like objects (filled and open circles) and AST stars (grey dots). Filled circles mark 128 star-like objects selected for further analysis, and open circles denote extended objects that were omitted from the analysis.

(in any filter) larger than 0.3 mag. Then we visually inspected the measured objects in the images and rejected those that lay on the obvious background galaxies, bright MW stars, or large artefacts. This left us with $N=756$ objects. Sharpness and photometric errors of these objects in the $F 475 W$ and $F 814 W$ HST WFC3 passbands are shown in Fig. 2a-d.

Star detection and photometric accuracy depend on image resolution, PSF quality, the object signal-to-noise ratio $(\mathrm{S} / \mathrm{N})$, and field crowding. A proper assessment of the photometric completeness and biases is crucial for a reliable CMD interpretation even of a single stellar population objects; see, e.g. Stonkute et al. (2008). An artificial star test (AST) is the best method to evaluate photometric errors and observation completeness. We used the DOLPHOT fake star program to generate a list of 35000 artificial stars, which were distributed evenly in the original images. The input colours and magnitudes of the artificial stars covered the complete range of the observed colours and magnitudes. The artificial stars sampled the colour $-1<(F 475 W-F 814 W)<5$ and magnitude $22<F 475 W<32$ ranges.

In order to optimise parameters for the photometry, we performed numerous tests with various source detection thresholds (SigFind and SigFinal) and a minimum allowed separation for two stars (RCombine) values. The best quality of photometry was achieved with SigFind $=4$, SigFinal $=$ 4 , and RCombine $=1.4$. These parameter values were applied for the final photometry and AST procedures. Completeness $\left(N_{\mathrm{r}} / N_{\mathrm{i}} \times 100 \%\right.$, where $N_{\mathrm{i}}$ and $N_{\mathrm{r}}$ are numbers of input and recovered artificial stars, respectively) estimates as a function of the $F 475 \mathrm{~W}$ and $F 814 W$ passband input magnitudes are shown in Fig. 2e and $\mathrm{f}$.

\section{Results and discussion}

The first step of the stellar population analysis within the observed field was to pre-select probable stars out of the 756

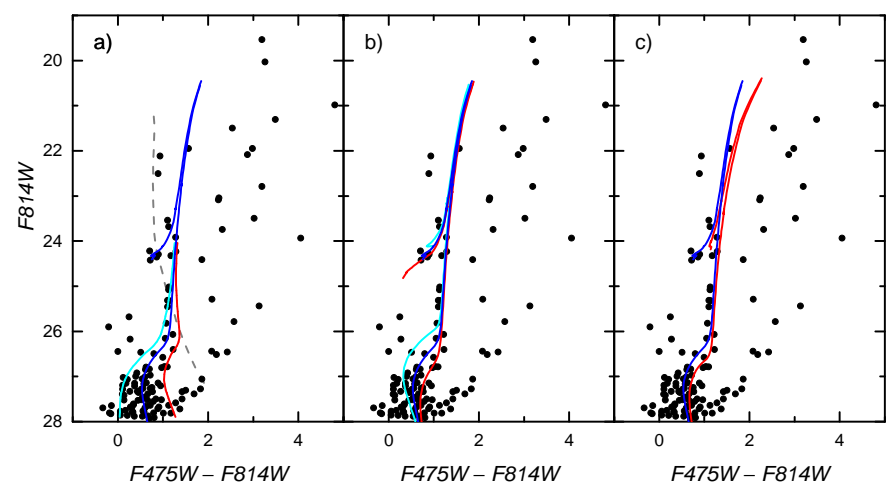

Fig. 4. CMDs of star-like objects in the studied field. An isochrone of $7 \mathrm{Gyr}$ age and $Z=0.0001$ metallicity (the blue line) is plotted in all panels. Additionally, we plot $a$ ) photometric AST star scatter limits (based on Fig. 2c and d), shown by cyan and red lines, as well as the lower limit of the MW foreground star distribution derived from the Besançon models, shown with the grey dashed line; $b$ ) isochrones of 5 and $10 \mathrm{Gyr}$ $(Z=0.0001)$ shown with cyan and red lines, respectively; and $c)$ an isochrone of $7 \mathrm{Gyr}$ and $Z=0.0006$ shown with the red line.

measured objects. In order to clean out the stellar population from extended objects, we used the sharpness ${ }_{F 814 W}$ vs. sharpness $_{F 457 W}$ diagram (Fig. 3) that was constructed for the AST and real stars. Following the DOLPHOT prescription (Dolphin 2000, see Eq. (14)), we rejected diffuse sources that have overly negative sharpness in both passbands and are therefore likely background galaxies or unresolved blends of stars. We determined limiting criteria for star selection based on sharpness vs. magnitude diagrams (Fig. 2a and b) of real and AST stars by taking a $3 \sigma$ criterion of sharpness scatter of the measured objects at $F 475 W=27.0$ and $F 814 W=25.5$, where the scatter of the AST star sharpness starts to overlap with the sharpness of objects that can be recognized by eye (sharpness $_{F 457 W}>-0.099$; sharpness s $_{F 14 W}>-0.078$ ). Additionally, we cut out a corner with a straight line connecting the points sharpness $s_{F 457 W}=-0.099$, sharpness $_{F 814 W}=0.0$ and sharpness ${ }_{F 457 W}=0.0$, and sharpness ${ }_{F 814 W}=-0.078$ (Fig. 3). As a result of this selection, we ended up with 128 probable star-like objects. However, some of these objects could still be unresolved galaxies, mainly the so-called "faint blue galaxies" that are abundant below $F 814 W \sim 25$ (Ellis 1997), Leo A blue stragglers (Momany 2015), or MW white dwarfs.

The colour-magnitude diagrams of the 128 star-like objects selected in the studied field are shown in Fig. 4. The PARSEC isochrone (Bressan et al. 2012) of $7 \mathrm{Gyr}$ age and $Z=0.0001$ metallicity (the blue line) is plotted in all panels. The isochrones in Figs. 4 and 5 are shifted assuming the Leo A distance modulus of 24.51 (Dolphin et al. 2002) and reddened taking into account only the foreground MW extinction, $A(F 475 W)=0.067$ and $A(F 814 W)=0.032$. In Fig. 4a we show photometric scatter limits with cyan and red lines, which are determined from the AST star distributions (Figs. 2c and d). In order to demonstrate age and metallicity effects, we plot the isochrones of 5 and 10 Gyr $(Z=0.0001)$ shown with cyan and red lines, respectively (Fig. 4b), and an isochrone of $7 \mathrm{Gyr}$ and $Z=0.0006$ shown with the red line (Fig. 4c).

To account for CMD contamination with MW stars, we produced a synthetic CMD in the direction of the Leo A galaxy by using the Besançon models (Robin et al. 2003). The grey dashed line (Fig. 4a) shows the lower limit of the MW foreground star distribution assuming the MW halo extension (extremely large) 


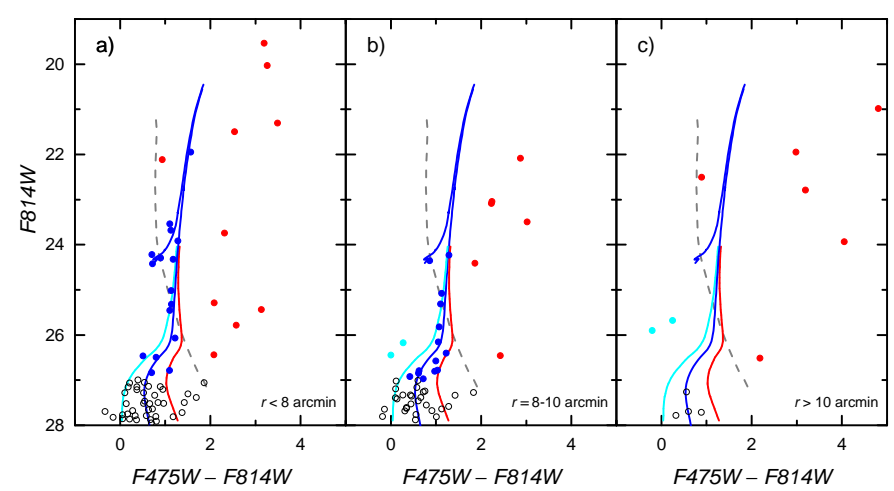

Fig. 5. CMDs of star-like objects in the studied field. The isochrone of 7 Gyr age and $Z=0.0001$ metallicity (the blue line), the limit of the MW stars as in Fig. 4a (the grey dashed line), and photometric scatter limits as in Fig. 4a (cyan and red lines) are plotted in all panels. The objects within radial distances of $\left.a) r<8^{\prime}, b\right) 8^{\prime}<r<10^{\prime}$, and c) $r>10^{\prime}$ from the galaxy centre are shown with circles in the corresponding panels. Filled colour circles mark the objects: in the vicinity of the isochrone lie probable Leo A members (blue); far from the isochrone and inside the CMD area occupied by MW stars (red); far from the isochrone lie probable compact faint blue galaxies, Leo A blue stragglers, or MW white dwarfs (cyan). Open grey circles mark faint star-like objects that were not used for the analysis.

of up to a $100 \mathrm{kpc}$ distance. Based on the synthetic CMD of MW stars, we assume that the star-like objects in Fig. 4 that are redder than $F 475 W-F 814 W \sim 2.0$ mainly belong to the MW galaxy.

For the analysis we selected only star-like objects with a high photometric accuracy, $F 814 W<27$. In order to trace stellar populations in the Leo A outer regions, we decided to employ RGB as well as horizontal and subgiant branch stars. From the isochrone of $7 \mathrm{Gyr}$ age and $Z=0.0001$ metallicity, we estimated an expected number ratio of stars along these sequences in the magnitude ranges of $24.5<F 814 W<27.0$ and $20.5<F 814 W<24.5$ and found a very good agreement with the observed star number ratio. This test suggested that the overdensity of stars along the isochrone seen in the CMD (Fig. 4) can be attributed to the Leo A galaxy stellar population.

In Fig. 5 the filled circles mark the objects that are probable Leo A members (blue), probable MW stars (red), probable compact faint blue galaxies, Leo A blue stragglers, or MW white dwarfs (cyan). Open grey circles mark faint star-like objects that we did not use for the analysis. An isochrone of 7 Gyr age and $Z=0.0001$ metallicity (the blue line), photometric scatter limits as in Fig. 4a (cyan and red lines), and the limit of the MW stars as in Fig. 4a (the grey dashed line) are plotted in all panels of Fig. 5. The panels in Fig. 5 show objects located at various distances from the galaxy centre: (a) $r<8^{\prime}$, (b) $8^{\prime}<r<10^{\prime}$, and (c) $r>10^{\prime}$. These objects are marked in Fig. 6 with the same colours.

In order to roughly estimate the age of stellar populations, we used the PARSEC isochrones, release v1.2S (Bressan et al. 2012) of $Z=0.0001-0.001$ metallicity. We found the best match of the isochrones with the distribution of star-like objects in the CMD in the case of the lowest available metallicity, $Z=0.0001$. Based on the CMD fitting with isochrones, we find old ( $>5$ Gyr) stellar populations of very low metallicity $(Z \sim 0.0001)$. However, the application of lower-metallicity isochrones would lead to an older age of stellar populations. On the other hand, the absence of stars on the blue horizontal branch would contradict this conclusion.

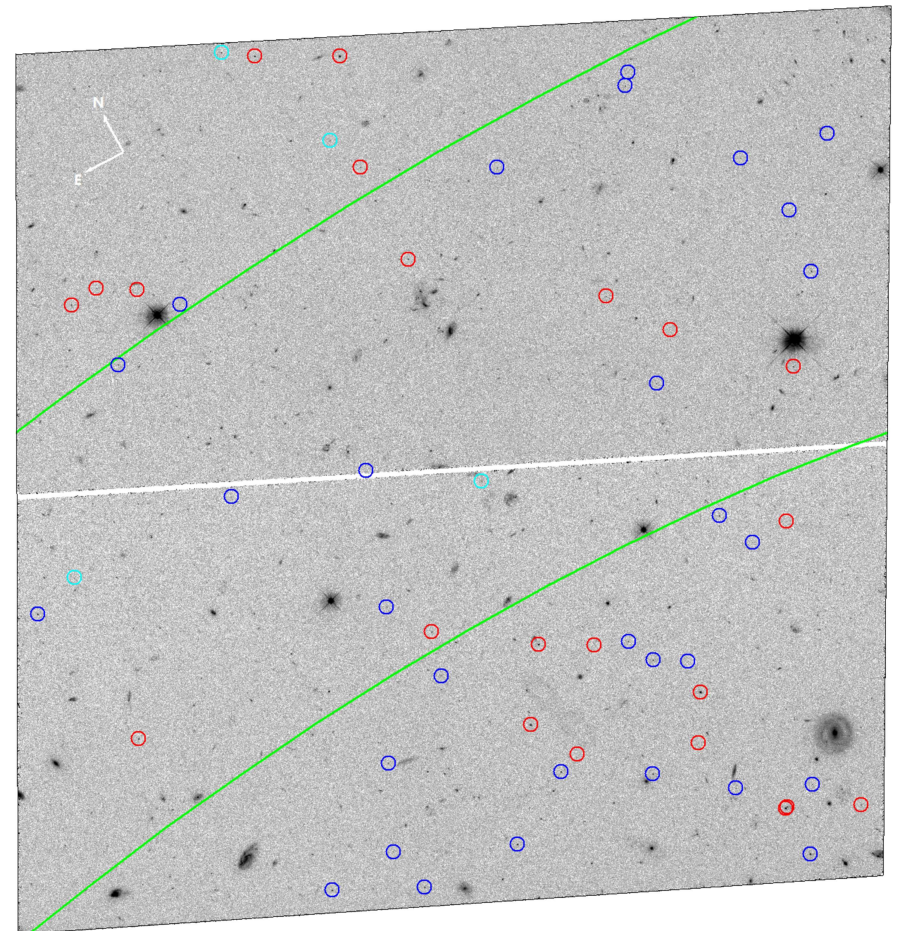

Fig. 6. Distribution of star-like objects in the studied field. The colourcoding is the same as in Fig. 5. Segments of the ellipses centred on Leo A of $a=8^{\prime}$ and $10^{\prime}\left(b / a=0.6\right.$; PA $\left.=114^{\circ}\right)$ are overplotted. Northeast directions are shown by white arrows.

A distribution of star-like objects in the studied field is shown in Fig. 6. Segments of the ellipses (green) centred on Leo A of $a=8^{\prime}$ and $10^{\prime}\left(b / a=0.6 ; \mathrm{PA}=114^{\circ}\right)$ are overplotted. Therefore, at least up to $a=10^{\prime}$, we see probable members of the Leo A galaxy. This conclusion fits the finding by Dolphin et al. (2002) well that RR Lyr C1-V01 is residing close to the ellipse of $a=10^{\prime}$ (Fig. 1). It is also important to note that we found that the stellar populations of Leo A are distributed more widely than the envelope of H I (Young \& Lo 1996; Hunter et al. 2012). This finding is important in the context of the discussion of an extremely gas-rich dwarf galaxy evolution scenario: inside-out, or outside-in.

\section{Conclusions}

We have performed photometry in the HST WFC3 field, which is located at the very outskirts of the Leo A galaxy and discovered that its stellar halo extends far beyond the previously known limits (Vansevičius et al. 2004). We stress, however, that the presence of a candidate RR Lyr star located at a distance of 5.9' from the galaxy centre (Dolphin et al. 2002) also provides evidence for a large halo of Leo A.

The detection of the outer halo stellar populations is based on the CMD analysis below the horizontal branch. We can currently assume that the semi-major axis of the stellar halo is $a \sim 2.3 \mathrm{kpc}$ $\left(a \sim 10^{\prime}, b / a=0.6\right)$. In order to establish the edge of the Leo A halo more clearly, we need to study a much larger field or to obtain accurate photometry for objects of $F 814 W>27$. However, even a small single HST WFC3 field that includes subgiant stars suggests the galaxy size (stellar component) to be larger by about one-third than previously estimated. Based on the CMD fit with isochrones (Bressan et al. 2012), we find old ( $>5$ Gyr) stellar populations of very low metallicity $(Z \sim 0.0001)$. However, 
to strengthen this conclusion, extremely deep, currently not feasible spectroscopic observations are needed.

Acknowledgements. The research has made use of the SAOImage DS9, developed by Smithsonian Astrophysical Observatory. The data presented in this paper were obtained from the Multimission Archive at the Space Telescope Science Institute. This research was funded by a grant (No. LAT-09/2016) from the Research Council of Lithuania.

\section{References}

Bernard, E. J., Monelli, M., Gallart, C., et al. 2013, MNRAS, 432, 3047 Bressan, A., Marigo, P., Girardi, L., et al. 2012, MNRAS, 427, 127 Brown, W. R., Geller, M. J., Kenyon, S. J., \& Kurtz, M. J. 2007, ApJ, 666, 231

Cole, A. A., Skillman, E. D., Tolstoy, E., et al. 2007, ApJ, 659, L17

Dolphin, A. E. 2000, PASP, 112, 1383

Dolphin, A. E., Saha, A., Claver, J., et al. 2002, AJ, 123, 3154

Ellis, R. S. 1997, ARA\&A, 35, 389

Fitzpatrick, E. L. 1999, PASP, 111, 63
Gil de Paz, A., Knapen, J. H., \& Lee, J. C. 2017, Formation and Evolution of Galaxy Outskirts (Cambridge: Cambridge University Press), Proc. IAU Symp., 321

Hunter, D. A., Ficut-Vicas, D., Ashley, T., et al. 2012, AJ, 144, 134

Kirby, E. N., Rizzi, L., Held, E. V., et al. 2017, ApJ, 834, 9

Knapen, J. H., Lee, J. C., \& Gil de Paz, A. 2017, Outskirts of Galaxies (Springer Int. Publ. AG), Astrophys. Space Sci. Lib., 434

Mateo, M. L. 1998, ARA\&A, 36, 435

Momany, Y. 2015, in Ecology of Blue Straggler Stars, eds. H. Boffin, G. Carraro, \& G. Beccari (Berlin, Heidelberg: Springer), Astrophys. Space Sci. Lib., 413,129

Robin, A. C., Reylé, C., Derrière, S., \& Picaud, S. 2003, A\&A, 409, 523

Schlafly, E. F., \& Finkbeiner, D. P. 2011, ApJ, 737, 103

Schulte-Ladbeck, R. E., Hopp, U., Drozdovsky, I. O., Greggio, L., \& Crone, M. M. 2002, AJ, 124, 896

Stonkutè, R., Vansevičius, V., Arimoto, N., et al. 2008, AJ, 135, 1482

Stonkutè, R., Arimoto, N., Hasegawa, T., et al. 2014, ApJS, 214, 19

Tolstoy, E., Gallagher, J. S., Cole, A. A., et al. 1998, AJ, 116, 1244

Tosi, M. 2003, Ap\&SS, 284, 651

van Zee, L., Skillman, E. D., \& Haynes, M. P. 2006, ApJ, 637, 269

Vansevičius, V., Arimoto, N., Hasegawa, T., et al. 2004, ApJ, 611, L93

Young, L. M., \& Lo, K. Y. 1996, ApJ, 462, 203 